\title{
Primary Familial Congenital Erythrocytosis: two novel EPOR mutations found in Spain
}

Bento C, Almeida H, Fernandez-Lago C, Ribeiro ML

\begin{abstract}
We present the study of two different families from Spain with primary familial congenital polycythemia (PFCP). Secondary causes of erythrocytosis were already excluded as well as JAK2V617F and JAK2exon 12 mutations. Sequencing analysis of the EPOR gene detected two novel heterozygous mutations. One with autosomal dominant transmission (c.1311_1312delTC), the other found in only one individual with unknown familial history (c. $1235 \mathrm{C}>\mathrm{A}$ ). The type and location of the mutations suggest that they can be the cause of the erythrocytosis observed in these patients.
\end{abstract}

\section{Introduction}

Congenital erythrocytosis is a rare disorder where patients present with high hemoglobin levels without a secondary cause. Knowledge about the molecular mechanisms involved in familial erythrocytosis has improved in recent years (Patnaik 2009).

Taking into account the hematological parameters, familial history and serum erythropoietin (Epo) levels it is possible to establish a diagnostic algorithm (McMullin 2005). Low serum Epo level with a strong familial history can be due to mutations in Epo receptor gene (EPOR), known as Primary familial congenital polycythemia (PFCP)(Kralovics 2001). Identification of the causal mutations in congenital erythrocytosis helps to establish the clinical diagnosis and treatment, however cases with mutations identified are sparse and the underlying molecular defect remains unknown in the great majority of described cases.

\section{Clinical Cases}

We studied two different cases from Spain referred to our laboratory for investigation of erythrocytosis with normal leukocyte and platelet counts and no splenomegaly. All secondary causes causing increased hemoglobin levels were already excluded as well as the JAK2 V617F and exon12 mutations. All subjects studied gave their informed consent.

\section{Case 1}

Three members of the same family, belonging to three different subsequent generations, from La Coruña, Spain.

The propositus is a 69-year-old man under regular phlebotomy treatment, diagnosed 14 years ago with idiopatic erythrocytosis. His son (42-years-old) and his grandnephew (3years-old), presented also with high hemoglobin levels (Hb 18.9 and $20.0 \mathrm{~g} / \mathrm{dL}$, respectively) All of them have serum Epo bellow $2.0 \mathrm{mU} / \mathrm{mL}$ (Normal $3-34 \mathrm{mU} / \mathrm{mL}$ ).

Since the Epo levels were low, and the family history suggests a dominant inheritance pattern, we sequenced the EPOR gene and found a novel heterozygous frameshift mutation due to the deletion of two nucleotides (c.1311_1312delTC) (Figure 1A).

This mutation results in a premature termination at codon 443 which predicts the production of an EPOR molecule truncated for 66 amino acids at the C-terminal end of the protein (p.Pro438Metfs*6)(Figure 1C)

\section{Case 2}

A 73-year-old male from La Coruña, Spain, presented with Hb18,9 g/dL; hematocrit (Hct) $58 \%$. Clinical examination was unremarkable, with no signs or symptoms of respiratory insufficiency. Serum erythropoietin level was markedly reduced $(2.9 \mathrm{mIU} / \mathrm{mL})$.

Neither children nor other family members were available to be studied. 
Sequencing analysis of the EPOR gene revealed a nonsense mutations (c.1235C >A) (Figure 1B). The mutation creates a premature stop codon (p.Ser412*) predicting the production of an EPOR molecule truncated for the last 97 amino acids at the C-terminal end of the molecule (Figure 1C).

In both cases mutations in $H B B, H B A, H I F 2 \alpha$ (exon12), DPGM, VHL or PHD2 genes were excluded by direct sequencing of all the exons and exon-intron boundary sequences

\section{Conclusions}

We present the study of two different Spanish families with congenital erythrocytosis. High hemoglobin and hematocrit levels are the only phenotypic manifestation of the disease. Sequencing analysis of the EPOR gene detected two novel heterozygous mutations. One was found in three members of the some family associated with autosomal dominant transmission, the other was found in only one individual, familial history was unknown.

According to the PubMed and data registered in Leiden Open-Access Variation Database (LOVD) (Fokkema 2005) the mutations detected were not yet described in the literature. Another EPOR mutation associated with congenital erythrocytosis, c.1317G $>$ A [p.(Trp439*)] have already been described in the Spanish population (Rives et al. 2007). Since first description of EPOR mutations associated with PFCP (de la Chapelle 1993) fourteen new mutations have been described. They are nonsense or frameshift mutations leading to cytoplasmic truncation of the Epo receptor, resulting in a loss of the C-terminal negative regulatory domain. This domain contains the tyrosine (Tyr) residues (Tyr426, Tyr454 and Tyr456) responsible to down-regulate the Epo bounding to EpoR via endocytosis and degradation mechanism (D'Andrea 1991). The mutations found in this study lead to a truncated receptor where two (p.Pro438Metfs*6) or three (p.Ser412*) of these tyrosine residues were lost (Figure $1 \mathrm{C}$ ).

Although functional studies were not made in order to confirm the pathogenic role of these mutations the type and location of the mutations suggest that they can be the cause of the erythrocytosis observed in these patients. Mutations in the other genes described as associated with erythrocytosis were excluded.

\section{References}

D'Andrea AD, Yoshimura A, Youssoufian H, Zon LI, Koo JW, Lodish HF (1991) The cytoplasmic region of the erythropoietin receptor contains non overlapping positive and negative growth-regulatory domains. Molecular and Cellular Biology 11: 1980-1987. de la Chapelle A, Träskelin AL, Juvonen E. (1993) Truncated erythropoietin receptor causes dominantly inherited benign human erythrocytosis. Proceedings of the National Academy of Sciences 90(10): 4495-4499.

Fokkema IF, den Dunnen JT and Taschner PE (2005) LOVD: easy creation of a locusspecific sequence variation database using an "LSDB-in-a-box" approach. Human Mutations 26: 63-68.

McMullin MF, Bareford D, Campbell P, Green AR, Harrison C, Hunt B, Oscier D et al. (2005) Guidelines for the diagnosis, investigation and management of polycythaemia/erythrocytosis. British Journal of Haematology 130(2): 174-195.

Patnaik MM, and Tefferi A (2009) The complete evaluation of erythrocytosis: congenital and acquired. Leukemia 23(5): 834-844.

Kralovics R, Prchal JT (2001) Genetic heterogeneity of primary familial and congenital polycythemia. American Journal of Hematology 68:115-121. 
Rives S, Pahl HL, Florensa L, Bellosillo B, Neusuess A, Estella J, Debatin K M, et al. (2007) Molecular genetic analyses in familial and sporadic congenital primary erythrocytosis. Haematologica 92(05): 674-677.

\section{Captations}

Fig.1 DNA sequence of a segment of the exon 8 of the EPOR gene showing the c.1311_1312delTC (A.) and the c.1235C >A (B.) mutations. C. Alignment of wild-type and mutant amino acids sequences, with tyrosine residues $(\mathrm{Y})$ underlined. 